\title{
Semi Automatic Estimation and Visualization of Left Ventricle Volumes in Cardiac MRI
}

\author{
MM García de Pablo ${ }^{1}$, N Malpica ${ }^{1}$, MJ Ledesma-Carbayo ${ }^{1}$, LJ Jiménez-Borreguero ${ }^{2}$, A Santos $^{1}$ \\ ${ }^{1}$ ETSI Telecomunicación, Universidad Politecnica Madrid, Spain \\ ${ }^{2}$ Hospital de la Princesa, Madrid, Spain
}

\begin{abstract}
The main goal of this work was to implement an easy and convenient new tool to load, visualize and segment Cardiac Magnetic Resonance Images (CMRI) in order to obtain basic parameters for cardiac function estimation and to visualize the results in a quantitative and graphical way. The proposed method tracks the contour of the left ventricle at endocardial level through all the cardiac cycle to measure the ventricular volume and the ejection fraction, using active contour techniques to differentiate structures within an image.
\end{abstract}

\section{Introduction}

MR images are very useful for the diagnosis of a great number of pathologies in all medicine fields but mainly in the identification of damages in the central nervous system, brain and medulla, and in the detection of muscle - skeleton irregularities. MRI is becoming increasingly important in cardiology, for the characterization and visualization of cardiac anatomy and function. Nowadays MRA (Magnetic Resonance Angiography) is used regularly in the visualization of arterial and vascular malformations, as well as thrombus or clots and in the evaluation of carotid arteries and brain irrigation. The images themselves provide a huge quantity of information; nevertheless it's through their analysis and quantification that it's possible to take a more based and accurate decision [1].

Quantitative measurements of ventricular volume allow the physician to have a glance at the anatomy of the ventricles, and to evaluate the consequences of myocardial infarction, the possible myocardiopathys, as dilated, hypertrophic etc. Moreover there are very relevant parameters such as the ejection fraction $(\mathrm{EF})$ to evaluate the global function of the heart. The EF is the proportion of blood ejected by the heart in each cardiac contraction or beat. A low EF indicates the weakening of the cardiac pump, and this is associated with worse prognosis and with high risk at short term of Sudden Cardiac Death (SCD) [2].
The ejection fraction is defined as the normalized difference between the ventricular volume at the end of systole (ESV) and at the end of diastole (EDV).

$\mathrm{EF}=(\mathrm{EDV}-\mathrm{ESV}) / \mathrm{EDV}$

These parameters are essential not only for diagnosing a cardiac disease but for evaluating its progression with time. As an example, an irregularity in the anatomy is usually followed by a dysfunction of the cardiac pump. The analysis of ventricular function using MRI is very useful to select patients for clinical or resynchronization therapy, and to make the follow up of clinical therapy.

The EF is usually calculated with 2D-Echo, but this technique cannot provide a measure as accurate as MRI does (MRI 3D), mainly because the different tissues are not so easily identifiable. The values obtained are acceptable to estimate the functioning of any heart, but in order to evaluate the progression of the disease or the improvement achieved with a particular therapy the measure should be more accurate [3].

Until now, commercially available implemented tools are not fully automatic. The specialist should outline the contour of the endocardium in each image to obtain the value of the area contained inside. If we take into account that a complete sequence of images of the heart is a 4 dimensional matrix involving around 10 slices and 20 time frames per slice, the specialist should outline accurately around 200 images, which it's not only very time consuming but also observer dependent.

Our aim has been to develop a tool to simplify this procedure, reducing the time employed in each study. It's based on a semi-automatic method that allows the user to initialize just one of the images of each slice of the heart. The initialization is not required to be very accurate, since the active contour algorithm will detect the exact limit of the structure.

Once the contour has evolved to the real outline, it measures the area trapped inside. As the DICOM images contain all information about pixel size and inter-slice distance, the tool can integrate the measured areas to obtain the ventricular volume in each frame of time during the cycle, and to represent it the volume graphically. 


\section{Materials and methods}

The proposed segmentation algorithm is based on active contour models [4], which are controlled by a combination of different energies trying to place them over the desired region of an image. The novelty of this method is the use of energies not only obtained from the image, but also from the external (a priori) knowledge of the desired structure. From the image, intensity, gradient or texture energies can be obtained. Geometric criteria can be included as "a priori" knowledge to limit the contour to conform to some sizes or shapes or to move only in some specific areas of the image. In this work three kinds of energies have been used: internal, external and obtained from the image. The aim of the snake is to minimize the following energy functional.

$$
\begin{gathered}
\Phi: V(s) \Rightarrow \int_{0}^{l}\left\{E_{\text {int }}(V(s))+E_{\text {im }}(V(s))+E_{\text {ext }}(V(s))\right\} d s \\
\mathrm{~V}(\mathrm{~s})=[\mathrm{x}(\mathrm{s}), \mathrm{y}(\mathrm{s})] s \in[0,1]
\end{gathered}
$$

where $\mathrm{V}(\mathrm{s})$ represents the contour, as a series of pixels in the image.

In this case, the energies used to conform the contour are:

Internal: dependent only from the relative situation of the points.

- Elastic: to shrink the contour.

- Curvature: to smooth the contour.

- Continuity: the contour tends to a circle.

- Balloon: to expand or contract the contour.

Image: obtained from the properties of the image itself.

- Intensity: gray level of the image.

- Gradient: signs borders or discontinuities.

v External: represent the restriction imposed from the "a priori" knowledge.

In our method the "a priori" knowledge is not introduced as an external energy but through weight constants in the other energies directing them to conform a circular shape contour that shrinks continuously from one frame to the following.

The movement of the snake is guided by an energy optimization algorithm, which finds the minimal energy point around each point of the snake. This method is called Greedy algorithm and although it doesn't guarantee the global minimum for the image, it achieves good results in fewer time than the algorithms based in global minimums [5]. During the segmentation of a complete sequence, the number of points shaping the snake is allowed to vary, depending of its length in order to fit better with any of the contours. This is achieved by automatic insertion/deletion of control points to maintain a certain distance interval between neighboring points.
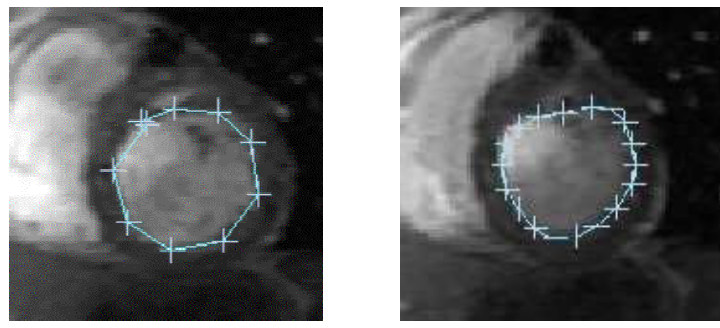

Figure 1. Initialization and segmentation of the first frame.

Following sections describe the different steps of the procedure.

\subsection{Initialization}

The segmentation process starts on the image acquired in diastole, by manual initialization of the contour by the user on this image. The specialist should place just 10 points around the endocardium of the left ventricle at the end diastolic image and the adjustment of the contour for the rest of the frames of this slice is automatic.

\subsection{Segmentation and propagation}

The algorithm uses the first initialization (ten points) to segment accurately the LV in that frame and the result is used to automatically initialize the next frame of the cycle. This transition can be performed either directly or after a correlation process between the two images. The results obtained with the correlation algorithm didn't differ significantly with the direct transition and the difference in time consumption was of the order of 10 to 1 , so we simply used the previous frame segmentation as the next initial contour. This option is in any case also implemented in our tool.

Traditional tracking schemes process the image sequences starting at end-diastole and progress throughout the whole cardiac cycle. However, these methods have many problems to keep the papillary muscles inside the ventricular volume coherently through the whole cycle. Main problems arouse because after maximum contraction, when the papillary muscles are attached to the myocardial wall, the active contour is not able to find again the myocardial borders and it gets trapped to the papillary muscles edges. In order to improve the results in slices where the papillary muscles are visible we propose a new tracking scheme as shown in figure 2. In this approach the tracking procedure starts at both ends of the sequence, therefore, the contour always shrinks from end diastole to end systole and the snake never collides with the papillary muscles. 


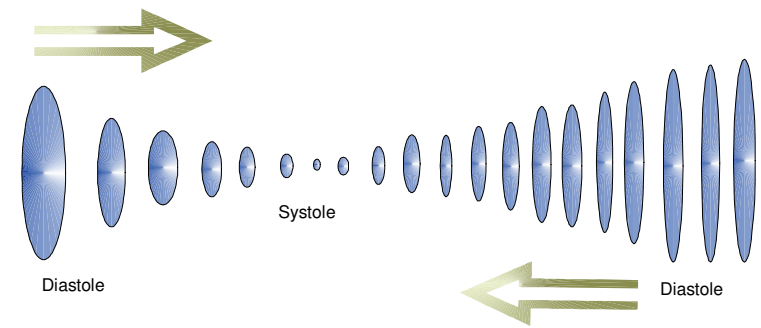

Figure 2 Order of the sequence to minimize papillary muscles to prevent the correct movement of the snake.

This procedure prevents the snake from getting trapped in the inner part of the ventricle because of the papillary muscles as it's shown in Figure 3.
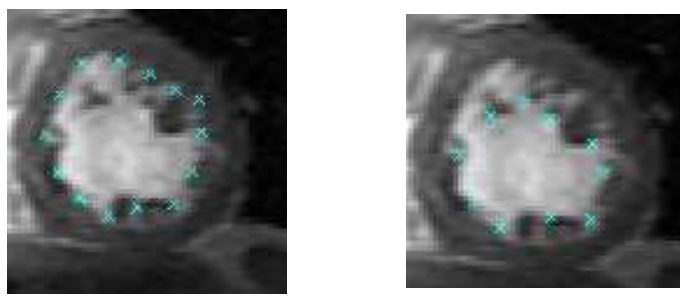

Figure. 3 Segmentation result including the papillary muscles (left) and not including them (right)

There is no agreement about the inclusion or exclusion of these muscles in the volume measurement. This algorithm includes them in all images, being consistent in the results.

Another option would be to exclude the papillary muscles volume from the commutation segmenting them muscles independently. Therefore only blood is considered in the volume measurements [6].

\subsection{Parameter estimation}

The ventricular volume in each frame is obtained integrating the areas obtained for all slices in the frame. The values of pixel size and inter-slice distance are extracted from the DICOM header. The EDV and the ESV are obtained taking into account the ECG. The EF is calculated from these parameters as explained in the introduction.

\subsection{Volume representation}

The segmentation result of each frame in each slice is saved in a 4-dimensional matrix as a series of 10 points distributed homogeneously along the snake. This matrix is the basis of the graphic representation of the ventricle's movement.
This technique is applied to the points belonging to each particular frame in all the considered slices, obtaining the 3D ventricle for each frame or moment in the cardiac cycle. Displaying all these volumes in their correct order we obtain a 3D simulation of the ventricular movement through the cardiac cycle. Figure 4 shows the $\mathrm{LV}$ in all the 10 odd frames along the cardiac cycle.
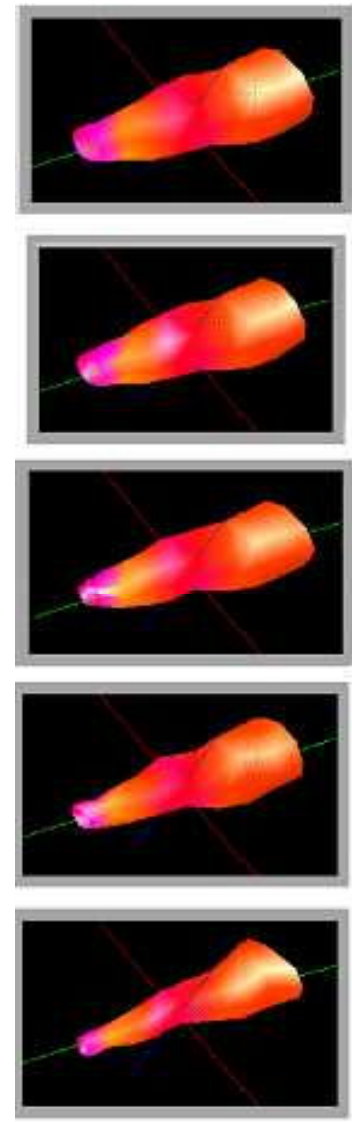
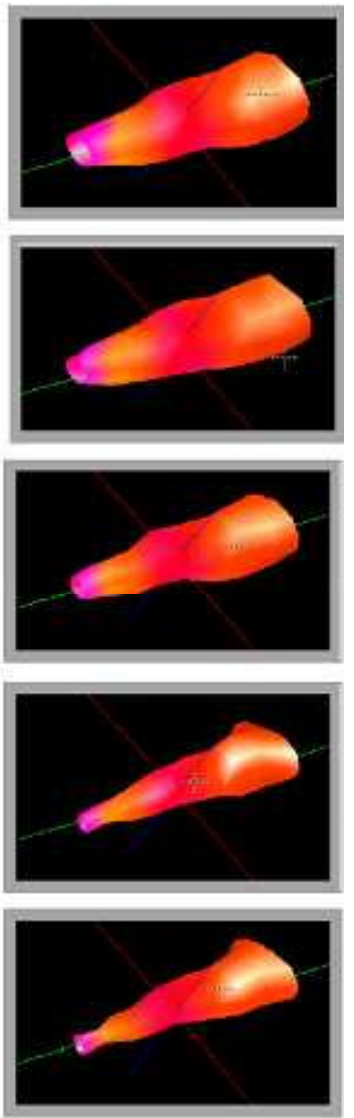

Figure 4. 3D representation of the $\mathrm{LV}$ in odd frames of the cardiac cycle.

Figure 5 shows the user interface of the software developed. The control blocks are placed in the right side of the screen. The first block allows the specialist to select the number of slices to be segmented. The second one is the Segmentation block. The initialization and the segmentation process are controlled from this menu. The option to correlate or not the frames is placed also in the segmentation block. The third block allows the specialist to review all the frames segmented in all the slices and to correct the result manually, saving the new contours. The last block starts the visualization tool and restarts the procedure. 


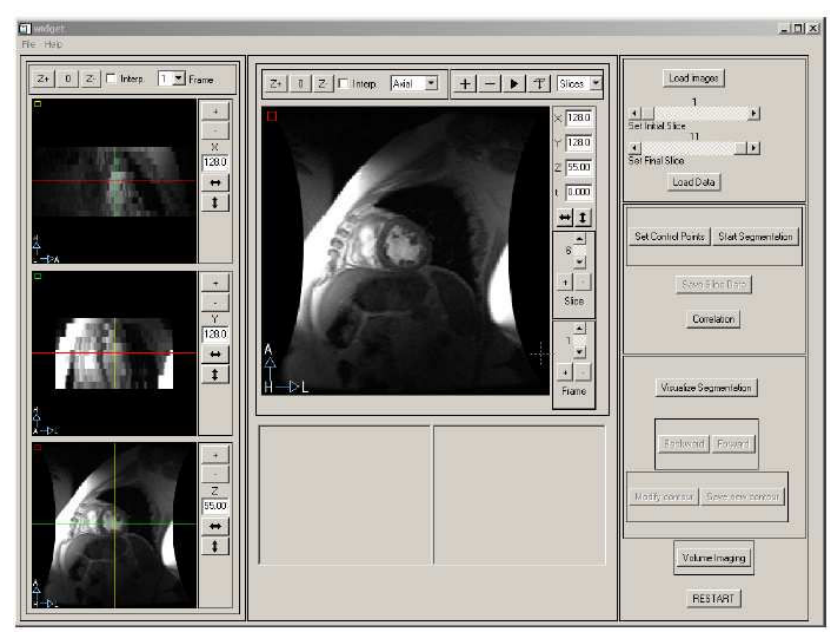

Figure 5. User interface of the application

\section{Results}

To evaluate the segmentation algorithm 6 Echo Gradient - Cine sequences were processed. They included normal and pathologic hearts (myocardial infarction and dilated cardiomyopathy). The results are presented in Table 1 and the normal ranges of these parameters are shown in Table 2.

Table 1. Parameters obtained from the 6 sequences

\begin{tabular}{|l|r|r|r|r|}
\hline & \multicolumn{1}{|l|}{ LVEDV } & LVESV & LVSV & EF \\
\hline STUDY 1 & 112.18 & 53.87 & 58.31 & 0.51 \\
\hline STUDY 2 & 132.51 & 54.47 & 78.04 & 0.58 \\
\hline STUDY 3 & 133.16 & 53.82 & 79.34 & 0.59 \\
\hline STUDY 4 & 110.67 & 38.68 & 71.99 & 0.65 \\
\hline STUDY 5 & 218.76 & 123.65 & 95.11 & 0.43 \\
\hline STUDY 6 & 131.32 & 54 & 77.32 & 0.58 \\
\hline
\end{tabular}

The evaluation of the algorithm was performed through the visual inspection of a specialist familiar with previous interactive tools. With the proposed software tool the manual modification of the contours was very infrequent and only in slices belonging to the base or to the apex. Results agreed with previous echocardiographic study and clinical status of the patients.

Table 2. Normal range of values for the parameters [7]

\begin{tabular}{|c|c|c|}
\hline PARAMETER & NORMAL RANGE & UNITY \\
\hline LVEDV & $125-135$ & $\mathrm{~m} \mathrm{I}$ \\
\hline LVESV & $55-65$ & $\mathrm{~m} \mathrm{I}$ \\
\hline SV & $\approx 70$ & $\mathrm{~m} \mathrm{I}$ \\
\hline EF & $0.5-0.6$ & \\
\hline
\end{tabular}

\section{Conclusions}

The developed tool provides a solution to the manual segmentation a 3D CMR study, minimizing the consumption of time of any specialist and providing acceptable results for ventricular volumes at different times during the cardiac cycle and for the ejection fraction of the LV. We have proposed a new tracking scheme that clearly avoids the possible inconsistencies in the segmentation due to the papillary muscles. The method provides ventricular volume computation and visualization during the whole cardiac cycle.

\section{Acknowledgments}

This work has been partially founded by the Spanish Health Ministry (Instituto de Salud Carlos III), research project PI041495 and Red Temática IM3 (G03/185)

\section{References}

[1] RadiologyInfo: the radiology Information resource for patients.Cardiac MRI (MRI of the heart, great vessels and adjacent structures)

http://www.radiologyinfo.org/content/mr_cardiac.htm

[2] Weber H, Allikmets K, Kornfeld G. Treating electrical instability in sudden cardiac death survivors - are we looking at the right side of the coin?. European Heart Journal 2004; 25:623-625.

[3] Rominger MB, Bachmann GF, Pabst W, Ricken WW, Dinkel HP, Rau WS. Left ventricular heart volume determination with fast MRI in breath holding technique: how different are quantitative heart catheter, quantitative MRI and visual echocardiography? Rofo. 2000 Jan;172(1):23-32

[4] Kass M, Witkin A, Terzopoulos D. Snakes: Active contour models. Int J Computer Vision 1987;1(4): 321-331.

[5] LaMacchia BA. Basis Reduction Algorithms and Subset Sum Problems, MSc Thesis, Dept. of Elect. Eng. and Comp. Sci., Massachusetts Institute of Technology, Cambridge, MA. 1991.

[6] Pluempitiwiriyawej C, Moura JMF, Wu YJL, Ho C“Cardiac MR Image Segmentation: Quality assessment of stacs". IEEE International Symposium on BioImaging, ISBI'04, Crystal City, VA, April 2004

[7] Pfisterer ME, Battler A, Zaret BL. Range of normal values for left and right ventricular ejection fraction at rest and during exercise assessed by radionuclide angiocardiography. Eur Heart J 1985;6(8): 647-655

Address for correspondence:

María J. Ledesma-Carbayo

Dept. Ingeniería Electrónica

ETSI Telecomunicación

Ciudad Universitaria sn

E-28040 Madrid (Spain)

mledesma@die.upm.es 